\title{
NRAS NM_002524.4:C.183A>C
}

National Cancer Institute

\section{Source}

National Cancer Institute. NRAS NM 002524.4:C.183A>C. NCI Thesaurus. Code C98443.

A nucleotide substitution at position 183 of the coding sequence of the NRAS gene where adenine has been mutated to cytosine. 